\title{
THE TRYPTIC ACTIVITY AND PRESENCE OF CORNIFIED SQUAMES IN MECONIUM AS A DIAGNOSTIC AID IN CONGENITAL INTESTINAL OBSTRUCTION
}

\author{
BY \\ JOHN L. EMERY \\ From the Department of Pathology, Children's Hospital, Sheffield
}

(RECEIVED FOR PUblication JUNe 20, 1951)

With the increasing efficiency of the surgery of the newborn the accurate diagnosis of congenital anomalies of the alimentary tract has become more important. The technical aids to the diagnosis of congenital intestinal obstruction are of three types: (1) radiological examination, taking advantage of the gas in the bowel and occasionally using opaque substances; (2) the examination of vomited and aspirated material for the presence of bile and pancreatic ferments; and (3) examination of meconium. Of these methods radiology is undoubtedly the most widely practised, and few clinicians use other tests. However, it is not an infallible guide as can be seen by the figures from the Children's Hospital in Boston in 1946, when only seven of 23 cases of congenital intestinal atresia were correctly diagnosed by radiology. The radiological appearances in intestinal anomalies are well described by Caffey (1950) and by Ladd and Gross (1941) and are not the concern of this paper.

The importance of the examination of vomited material and fluid obtained by gastric suction is obvious. The presence of bile indicates patency of the biliary and gastro-duodenal passages. If duodenal suction is used the findings of tryptic activity in alkaline fluid would suggest an absence of that type of meconium ileus which is usually associated with gross pancreatic change.

The naked eye examination of the stools in intestinal atresia is occasionally misleading. When the stool consists of a pale, mucoid thread or small green-centred fragments covered with mucus-like 'rabbit droppings' there is no confusion (Fig. 1), but not infrequently infants with atresia situated fairly high up in the intestinal tract pass meconium which is described by experienced paediatricians as being normal. It is these apparently normal stools which cause confusion in diagnosis and delay in treatment. This is doubly unfortunate since these high obstructions are most amenable to surgical treatment. In such cases more detailed examination of the meconium can be of assistance.

The test that is most widely known and used in

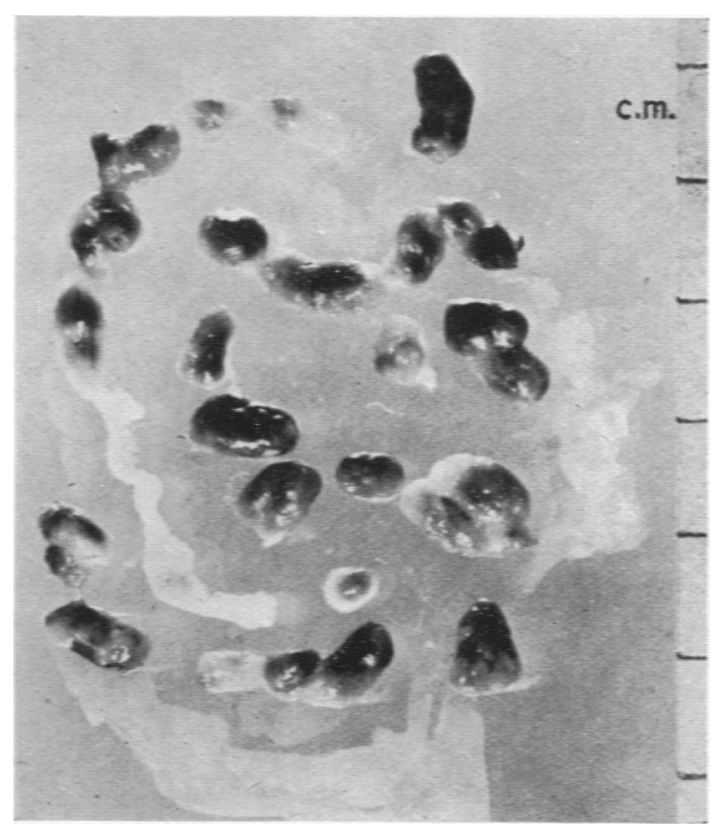

Fig. 1.-Abnormal meconium from a child with intestinal atresia, showing small green fragments, rabbit droppings ', covered with pale mucus and separated by threads of mucus.

the diagnosis of intestinal atresia was described by Farber in 1933. It depends upon the observation that normal meconium is formed, in part, from amniotic fluid swallowed in utero. This was clearly 


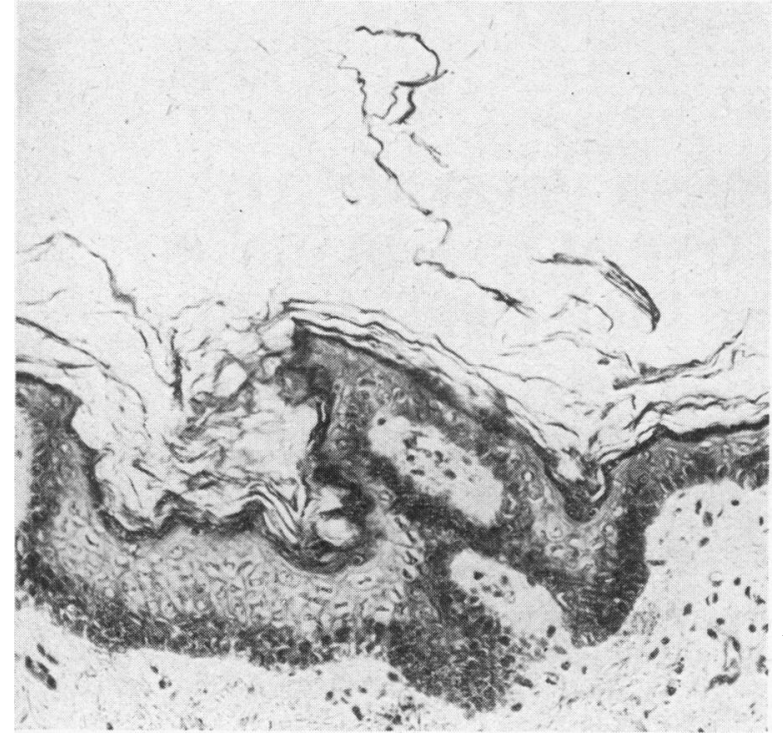

Fig. 2a.-Section of skin from newborn child showing cornified epithelial squames flaking off $(\times 150$ Masson's trichrome).

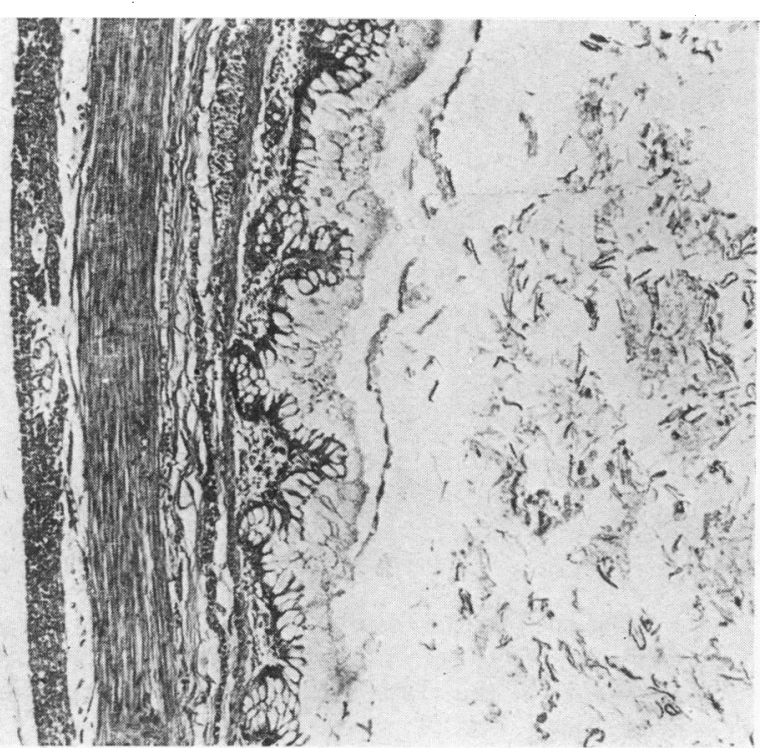

FIG. 2b.-Section of gut containing meconium from a newborn child showing the squames in the meconium ( $\times 100$ Masson's trichrome).

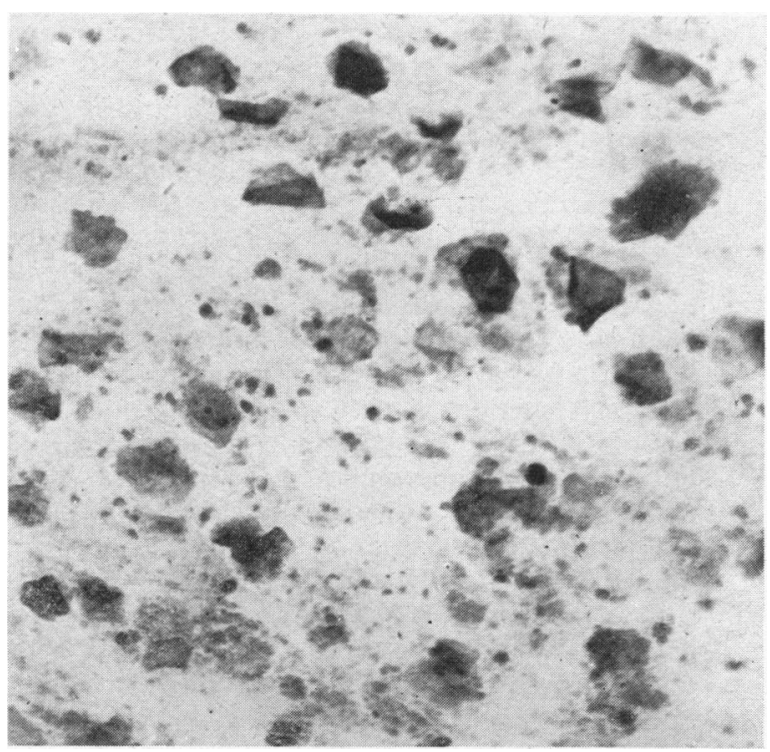

FIG. 2d.-Smear of meconium from a normal child showing cornified squames. $\times 200$. (For stain, see Appendix.) 
illustrated by Becker, Windle, Barth and Schulz (1940). They injected colloidal thorium hydroxide into the amniotic sacs of guinea-pigs and observed several complete cycles of passage through the intestinal tract in animals that were later born normally. Foetal ingestion and gastric concentration, though not complete intestinal cycles, have also been demonstrated in man. Amniotic fluid contains desquamated cornified epithelial cells and these, since they are not destroyed during passage through the alimentary tract, are normally present in meconium (Fig. 2). In Farber's test these cells are identified by their staining properties (for technical procedure see Appendix). Woody (1947), showing the usefulness of Farber's test, reported correct diagnosis with it in 23 of 24 instances in 1946-47, compared with a correct diagnosis in only seven of 23 cases in the previous nine years when the test was not used. The test is recommended in most of the standard textbooks of paediatrics. There are, however, some sources of error. Errors can occur in staining and in examining the films. Cornified squamous cells are present round the anus and become attached to the surface of the stool as it is passed. Squamous cells may also be introduced into the rectum by the examining finger or into the colon by an attempt at irrigation. For that reason Farber suggests that the meconium of which the film is made be taken from the inside of the meconium stool. The tenacious nature of the meconium makes this operation difficult, hence the finding of a few cornified squamous cells does not theoretically exclude the diagnosis of intestinal atresia.

During a survey in this laboratory of the tryptic activity of stools it was noted that there was tryptic activity in the first meconium stool of many infants, whereas none was found in two cases of meconium ileus and in two cases of intestinal atresia. For that reason a survey of the tryptic activity of meconium was carried out to test its usefulness in neonatal diagnosis, and, at the same time, the value of Farber`s test was checked.

\section{Materials and Methods}

The first, and in some cases subsequent, specimens of meconium were collected from 100 normal infants born at a municipal maternity hospital. Meconium was also obtained from three cases of meconium ileus and five of intestinal atresia, all cases being confirmed at necropsy or operation.

Meconium was collected by the nursing staff into small waxed containers. These specimens were examined separately for tryptic activity, and in 94 instances Farber's test was also carried out. The slides were examined by me for cornified cells and then sent, as a set of numbered slides, to three pathologists who consented to examine the smears and report on the presence or absence of cornified cells. The pathologists had no knowledge of the children from which the specimens came other than that some were normal and some abnormal, and were unaware of each other's findings. The slides were later decolorized and re-stained by a different method (Appendix), and again examined by other pathologists.

Tryptic activity was measured using a simplified modification of the method described by Andersen and Early (1942) for the examination of duodenal juice (Appendix).

\section{Results}

Tryptic Activity. The tryptic activity of 100 first specimens, together with 42 second specimens of meconium from 100 unselected normal infants, is expressed as a distribution curve in Fig. 3.

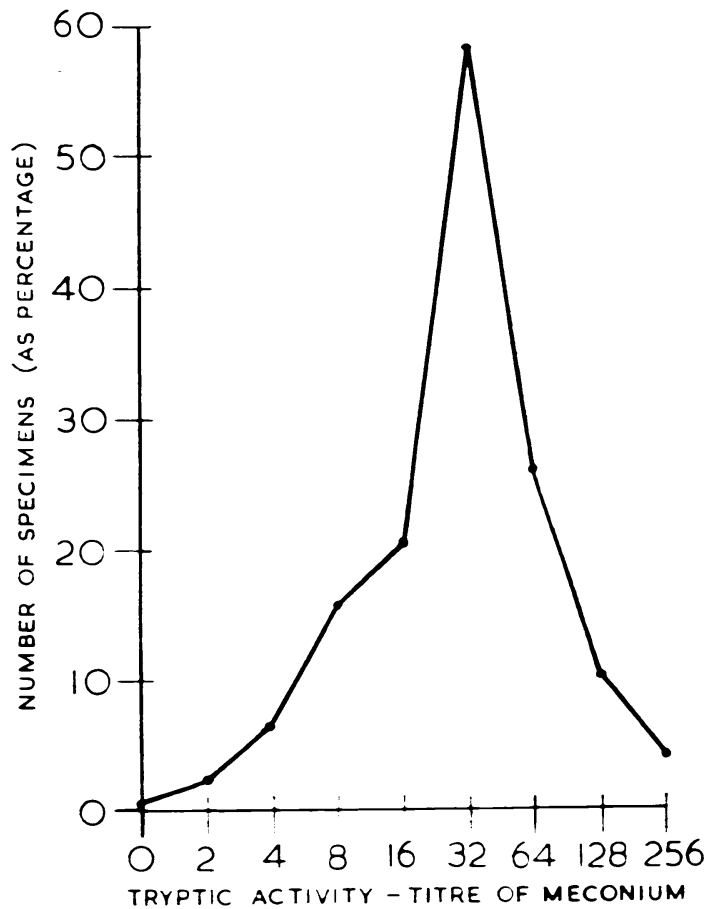

FIG. 3.-Distribution diagram showing the percentage of specimens with tryptic activity at a varying dilution; 142 specimens of meconium from 100 normal newborn children.

Estimations were at first carried out using four tubes only, i.e. to a dilution of 1 in 16 and to a final negative dilution in the last $\mathbf{5 0}$ specimens only.

One case showed no tryptic activity. Three out of $142(2.1 \%)$ had activity in only the first dilution; nine $(6 \cdot 3 \%)$ to second dilution and $23(15 \%)$ to the third dilution, i.e. one in eight. Fifty-eight out of 142 showed activity to a dilution of 1 in 16 , but since 
the majority of these had not been tested further only specimens examined to dilutions and showing no activity beyond this dilution were u sed in the distribution graph. Of the $\mathbf{5 0}$ specimens examined to further dilutions, $29(58 \%), 13(26 \%)$, five $(10 \%)$ and two $(4 \%)$ showed activity to the titre of 1 in 32 , 64,128 and 256 respectively.

We were unable to obtain further specimens from the one case showing no tryptic activity in the first specimen or from two of the three cases showing activity only in the first tube. Further specimens of meconium were available from the third child, and activity was present at a dilution of 1 in 4 when the child was 10 hours old, 1 in 32 at 20 hours and again at $\mathbf{4 0}$ hours.

Of the meconium from five cases of intestinal atresia, the material was green in four and showed no tryptic activity. In the fifth case the meconium was grossly abnormal consisting almost entirely of greyish mucus; tryptic activity occurred in the first tube only with this material.

Examination for Cornified Cells. Ninety-six numbered slides of meconium, four from cases of atresia and the rest obtained from normal newborn children, were stained using Farber's technique. The slides were stained to my satisfaction and were then sent to three pathologists who reported on them independently. Their results were: pathologist $\mathbf{A}$, 30 positive, 66 negative; pathologist $B, 31$ positive, 65 negative; pathologist C, 46 positive, 50 negative. Superficially there appeared to be some agreement, but, when the cases were analysed, it was found that frequently a slide which one had recorded as double positive was regarded as negative by another. In all, 14 slides only were consistently reported as positive and 38 as negative. Of the four smears from cases of atresia, two had consistent negative reports and the other two were reported as positive by at least one pathologist. Thus, of 96 slides the report was consistently correct clinically in only 16 instances. Complaints of the staining method were made by all. The chief complaints were of the variation of stain-retention of the squames, and the absence of stain in the rest of the tissue which made the preparations tiring to look at and difficult to keep in focus. The smears were therefore decolorized and re-stained using part of the usual Masson's trichrome stain (Appendix), as it had been noted that sections of the newborn intestine stained by the latter method showed a brilliant contrast between bright red squames and green background with this method. Squames stained in this way were much more easily identified and were found by my colleagues in all the smears which had previously been found negative.

Large numbers of squames were seen in the majority of the preparations, but in extremely small numbers in a few of the smears from normal meconium. In all of the cases of atresia a few squames were found. It was not found possible to distinguish the few normal smears with very few squames from the smears from the cases of atresia.

\section{Discussion}

The object of this study was to investigate the normal variation of tryptic content and cornified squames of meconium and so gauge their value in the diagnosis of atresia. One test depends on the infant swallowing amniotic fluid and the other on the secretion of trypsin by the pancreas. Both depend on the patency of the alimentary tract below the level of the duodenum, but the former also requires patency of the upper alimentary tract. The results show that Farber's test may give results which are misleading and also explain why in some centres the test is found useful, but in many instances only lip service is paid to the test. When examination for cornified squames is carried out by a clinician or general pathologist better results are likely if the smear used is stained with acid fuchsin and with light green as a counterstain instead of using the staining method recommended by Farber. There is no justification for statements such as that recently made by Wyatt (1951): "If an atresia is present, no meconium shows on the diaper, and the rectal swab gives a negative Farber test. If the condition is stenotic in nature small amounts of meconium are present and Farber's test is positive.' The conclusions that can be drawn from the investigation of Farber's test are that (1) it is not an 'all or none' test, (2) that when Farber's stain is used previous experience of the method makes a great difference to the result obtained, but this difficulty can to a great extent be overcome by using a different staining technique; (3) the failure to find cornified epithelial cells is not proof of atresia; (4) the presence of a few squames is not proof of patency; (5) the test would seem to have definite value in that the finding of many squames is an indication that a lumen is present throughout the alimentary tract.

There are embryological, clinical and pathological considerations in support of the use of tryptic activity as a measure of patency of the lower alimentary tract. There is little doubt that the pancreas secretes before birth; secretory granules are present in the pancreas of the foetus (Keene and Hewer, 1929), and increase greatly just before birth (Werner, 1948). Madey and Dancis (1949) showed that the concentration of proteolytic enzymes from the duodenum of premature infants is similar to that in older children, and Klumpp and Neale (1930) 
showed that the proteolytic enzymes in the newborn and the rest of childhood are equal.

The examination of meconium for tryptic activity is similar to the test widely used as an aid to diagnosis in fibrocystic disease of the pancreas (Shwachman, Patterson and Laguna, 1949). The digestion of the gelatine film on an $x$-ray plate is a suitable method of performing the test, but we have preferred to use a tube method as it is more convenient, and, in this laboratory, the same method is used for duodenal fluid. The test takes altogether about an hour and a half and can be done with the minimum of technical skill.

The range of tryptic activity found in meconium shows the type of distribution usual in physiological material. The results show that in over $90 \%$ of instances the normal meconium showed tryptic activity to a dilution of at least 1 in 8 . In no case of atresia below the ampulla of Vater has tryptic activity been found with a titre of that degree. In two cases of atresia in which only necropsy material was available tryptic activity was present in the upper dilated segment and absent in the contents of the lower bowel. In a recent case of atresia, which had bile in vomited material and no tryptic activity of the meconium, there was a rapid onset of tryptic activity in the stools when the obstruction was relieved by operation.

Tryptic activity was absent in the meconium in three cases of meconium ileus, a finding to be expected in the type of ileus associated with pancreatic changes described by Farber (1944), but it is to be noted that tryptic activity was present in the faeces of the case of meconium ileus reported by Hinden (1950).

The absence of tryptic activity does not provide us with a certain diagnostic test for atresia. There was one normal infant with no tryptic activity in the first specimen of meconium. This extreme variation of the normal must be remembered.

If there is an atresia above the ampulla, tryptic activity should be present in the meconium and its absence would suggest a second obstruction lower down in the alimentary tract.

Since the two tests, one for cornified squames and the other for tryptic activity, depend upon funda- mentally different processes the results of the two are likely to be more useful than that of only one.

\section{Summary}

It is again pointed out that children with congenital intestinal atresia can pass meconium which to the naked eye appears normal.

An attempt is made to assess the range of tryptic activity in normal meconium and to verify the basis of Farber's test.

It was found that in $92 \%$ of 142 specimens from 100 normal infants, tryptic activity was present in meconium to a dilution of at least 1 in 8 . In five cases of intestinal atresia, tryptic activity was absent in four and present at a dilution of 1 in 2 in one case.

Meconium smears from 92 normal and four children with congenital atresia were stained as recommended by Farber and sent to three general clinical pathologists. Their reports were consistent and correct in only 16 of the 96 cases. This was found to be largely due to staining difficulties, and a more contrasting stain showed cornified squames in all smears but varying greatly in quantity. Squames were also found in small numbers in the smears from cases of atresia. Farber's test modified in this way should only be considered positive if large numbers of cornified squames are found.

If tryptic activity is absent in meconium, atresia below the ampulla of Vater or meconium ileus is likely. Tryptic activity present at a dilution of 1 in 8 or more would appear to exclude atresia below the duodenum.

It is recommended that in cases when the diagnosis of atresia is in doubt the meconium be examined both for cornified cells and for tryptic activity. Since the two tests depend upon fundamentally different processes the results of the two are unlikely to be equivocal.

A technical appendix is included.

Thanks are most sincerely accorded to the nursing staff of Nether Edge Maternity Unit for collecting specimens of meconium, and to the pathologists who examined the meconium films, Drs. Edwards, Gatman, Harding and Whitely, also to Mr. Robert Zachary for his interest and help, and to Nigel Blumsum for his voluntary technical assistance. The photographs were taken by $\mathbf{A}$. K. Tunstall.

\section{A P P E N D I X}

Farber's Test

The following procedure was evolved for clinical use. Make a thin smear of meconium from the inside of the specimen on a slide. If no meconium is available a rectal swab may be used. Put the smear immediately, or when dry, in a flat dish containing ether to dissolve the fat. Dry in air. Stain for one minute with $1 \%$ gentian violet or $1^{\circ}{ }_{0}$ methyl violet. Wash in running water. Decolorize in acid alcohol $\left(5^{\circ} \% \mathrm{HCl}\right.$ in $64.0 \mathrm{P}$. alcohol). Finally wash in water, blot, dry and mount. (Cornified squames are stained blue to violet; the background is colourless.)

\section{Modified Stain for Cornified Squames}

The technique is as follows.

Make smear, remove fat and dry as in the above method. Stain for two minutes in Masson's Ponceau-acid 
fuchsin. Decolorize for 15 minutes in $1 \%$ phosphomolybdic acid. Counterstain for 15 seconds in $2 \%$ light green. Wash, dry and mount. Cornified cells stain red, and the background green.

\section{Estimation of Tryptic Activity}

Materials. The following are required: $5 \%$ sodium bicarbonate; 0.7 gelatine (prepared as in the AndersenEarly technique) made in bulk, and stored in medical flats; $37^{\circ} \mathrm{C}$. waterbaths; small test tubes; wide-mouthed Wright pipette (marked at about $1 \mathrm{ml}$.); rubber teat.

Method. Put the gelatine to melt under a hot water tap. Using the Wright pipette, put $5 \%$ bicarbonate to the mark into a series of nine tubes in a rack. This same pipette, with a volume to the same mark, is used in all the subsequent dilutions and measurements. Add $5 \%$ bicarbonate to the meconium and mix to reach a consistency that can just be drawn up to the marked volume in the pipette. Transfer this to the first of the tubes containing bicarbonate, and mix by drawing up and down into the pipette. Transfer aliquot portions to the next tube, mix, and carry serial dilutions to the eighth tube discarding the aliquot portion at the eighth tube. The ninth tube is a control. Using the same pipette after washing in hot water, transfer volumes of the melted gelatine to each tube. Mix, incubate in a water bath for one hour, then place in running cold water and read when the control tube is seen to be completely solidified. (The tubes are also placed in the refrigerator overnight and reread in the morning.) Liquefaction is seen by tilting the tubes.

\section{REFERENCES}

Andersen, D. H. and Early, H. V. (1942). Amer. J. Dis. Child., 63, 891 .

Becker, R. F., Windle, W. F., Barth, E. E. and Schulz, M. D. (1940). Surg. Gynec. Obstet., 70, 603.

Caffey, J. (1950). 'Pediatric X-ray Diagnosis,' 2nd ed. Chicago.

Farber, S. (1933). J. Amer. med. Ass., 100, 1753. (1944). J. Pediat., 24, 387.

Hinden, E. (1950). Archives of Disease in Childhood, $25,99$.

Keene, M. F. L. and Hewer, E. E. (1929). Lancet, 1, 767.

Klumpp, T. G. and Neale, A. V. (1930). Amer. J. Dis. Child., 40, 1215.

Ladd, W. E. and Gross, R. E. (1941). 'Abdominal Surgery of Infancy and Childhood.' Philadelphia.

Madey, S. and Dancis, J. (1949). Pediatrics, Springfield, $4,177$.

Shwachman, H., Patterson, P. R. and Laguna, J. (1949). Ibid., 4, 222.

Werner, B. (1948). Ann. paediatr., Basel, 170, 8.

Woody, N. C. (1947). N. Orleans med.' surg. J., 100, 273.

Wyatt, O. S. (1951). J. Amer. med. Ass., 146, 236. 